\title{
2016 Ad Hoc Reviewers
}

Emotion extends its thanks to the following individuals who contributed their time and expertise to the journal by serving as ad hoc reviewers for manuscripts submitted from November 2015 to November 2016.

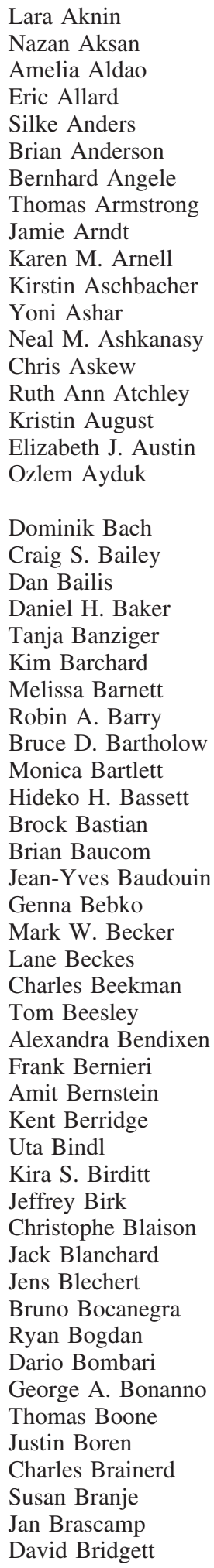

Margaret J. Briggs-Gowan

Pablo Briñol

Rebecca Brooker

Richard Brown

Stephanie Brown

Celia Brownell

Laura Brumariu

Greg Bryant

Brenda Bryant

Ross W. Buck

Andrew E. Budson

Jeni Burnette

Michael A. Busseri

Emily A. Butler

Lauren M. Bylsma

Manuel G. Calvo

Rafaela Campagnoli

Linda A. Camras

Turhan Canli

Rodrigo A. Cardenas

Cheryl Carmichael

Marisa Carrasco

Luis Carretié

Charles S. Carver

Salvatore Catanzaro

Anthony Cate

Justin Cavallo

Sarah Cavanagh

Sarah R. Cavanagh

Lisa Cavanaugh

Alice Chang

Tara Chaplin

Ian Charest

Jennifer Cheavens

Frances Chen

Wenfeng Chen

Andrey Chetverikov

Cecilia Cheung

Hae-Yoon Choi

Margaret S. Clark

Charles Clifton

Noga Cohen

Shana Cole

Paul Condon

Arin Connell

Tamlin Conner

Jonathan E. Cook

Alison Cooke

Olivier Corneille

Kathleen Corrigall

Jesse Cougle

L. E. Crawford

Hugo D. Critchley

Steve L. Crites

Eveline Crone

Michael Crowley

Robert A. Cummins
Kim M. Curby

Antonietta Curci

Melissa Cyders

Ljubica Damjanovic

James Danckert

Ellen de Brujin

Krista De Castella

Jan W. de Fockert

Jasper de Groot

Jozefien De Leersnyder

Andres De Los Reyes

Kirby Deater-Deckard

Andrew Delton

Ekaterina Denkova

Tracy A. Dennis

Bryan Denny

Nazanin Derakhshan

Birgit Derntl

Peter Descioli

C. Nathan DeWall

Leah R. Dickens

Manfred Diehl

Katherine Dixon-Gordon

Leah Doane

Helen Dodd

Florin Dolcos

Chris Donkin

Barney Dunn

C. Emily Durbin

Julie Earles

John D. Eastwood

Boris Egloff

Tsachi Ein-dor

Gillian Einstein

Nancy Eisenberg

Emily Elliott

Phoebe C. Ellsworth

Tammy English

Yasemin I. Erbas

Catherine Evers

Antonia Farmer

Katya Fernandez

José Miguel Fernandez-Dols

Rebecca Ferrer

Karen Fingerman

Johnny Fontaine

Gregory Fonzo

Jaclyn Ford

Brett Q. Ford

Dan Foti

Daryl Fougnie

Michael Frese

Paul Frewen

Jennifer M. B. Fugate

Michael Furr
Jeffrey Gagne

Isaac Galatzer-Levy

Xiaoqing Gao

Anne Gartner

Maria Gartstein

Karen Gasper

Kathleen Gates

Valeria Gazzola

Maria Gendron

Oliver Genschow

Amy Gentzler

Alexander L. Gerlach

Melissa Ghera

Eva Gilboa-Schechtman

Thomas Gilovich

Peter M. Gollwitzer

Sarah Gomillion

Amie M. Gordon

Alice Graham

Alicia A. Grandey

DeMond M. Grant

Kurt Gray

Katie Gray

Michael Greenstein

Christian Grillon

Jessica R. Grisham

Maud Grol

Igor Grossmann

Daniel Gruehn

Ingrid Haas

Brian W. Haas

Anke Haberkamp

Anneke Haddad

Stephan Hamann

Alfons O. Hamm

Jochim Hansen

Shlomo Hareli

Cindy Harmon-Jones

Paul L. Harris

Susan Harter

Jasmeet P. Hayes

Alexandre Heeren

Roelie J. Hempel

Hal E. Hershfield

Jari K. Hietanen

Gilad Hirschberger

Edward R. Hirt

Hidefumi Hitokoto

Sara D. Hodges

$\mathrm{Xu}$ Hong

Todd Horowitz

Gernot Horstmann

Marlies Houben

Kurt Hugenberg

Kathryn L. Humphreys

Jeffrey R. Huntsinger

Hyi Sung Hwang 
Alexandru Iordan

Muireann Irish

Jessica Irons

Derek M. Isaacowitz

Linda M. Isbell

Jeremy Jamieson

Carly Johnco

Dan R. Johnson

Rebecca Johnson

Iain T. Johnstone

Rafał Jończyk

Jutta Joormann

Yannick Joye

Joseph Kable

Lukasz Kaczmarek

Joscha Kaertner

Elise K. Kalokerinos

Emily Kappenman

Andreas Kappes

Mary Kayyal

Andreas Keil

Briana Kennedy

David A. Kenny

Sarah Kertz

Yoav Kessler

Anne Kever

Shari Kidwell

Elizabeth J. Kiel

Young-Hoon Kim

Michael A. Kisley

Shinobu Kitayama

Leonie Koban

Birgit Koopmann-Holm

Christoph Korn

Maria Kovacs

Michael W. Kraus

Anne Krendl

Mariska Kret

Assaf Kron

Mikko Lähteenmäki

Alan Lambert

Claus Lamm

Jens Lange

Shelby Langer

Carrie Langner

Stephen Langton

Regina Lapate

Jeff T. Larsen

Bonnie M. Le

Tae-ho Lee

Daniel H. Lee

Arnaud Leleu

Heather Lench

Tera D. Letzring

Sara M. Levens

Einat Levy-Gigi

Teresa M. Leyro

Ye Li

Maria Licata

Diane Lickenbrock
Belinda Liddell

Matt Lieberman

Daniel Lim

Cesar F. Lima

Tanja Lischetzke

Kimberly M. Livingstone

Sandra Llera

Marina Lopez-Sola

Elizabeth Losin

Jessica P. Lougheed

Peter Lovibond

Richard E. Lucas

Aaron Luebbe

Erika Lunkenheimer

Heather Macarthur

Carolyn MacCann

Laura MacPherson

Christopher R. Madan

Joe Magee

Sam Maglio

Alexandra Main

Matteo Malgaroli

Antony S. R. Manstead

Brett Marroquin

John Marsh

Nicole Martin

Cecilia Martinez-Torteya

Lynn Martire

Takahiko Masuda

Andrew M. Mathews

Michael Mathieu

David Matsumoto

Ethan McMahan

Dana L. McMakin

Kateri McRae

Eugene McSorley

Brian P. Meier

Thorsten Meiser

Peter Mende-Siedlecki

Daniel S. Messinger

Katherine Mickley Steinmetz

Eleanor Miles

Karen J. Mitchell

Yuri Miyamoto

Sanaz Mobasseri

Aprajita Mohanty

Joan Monin

Benoit Monin

Joann M. Montepare

Rani Moran

Jun Moriya

Natali Moyal

Manon Mulckhuyse

Nora A. Murphy

Greg Murray

Atsuko Nakagawa

Elizabeth Nawrot

Rob M. A. Nelissen

Nicole Nelson

Tricia Neppl

Steve Neuberg
Mark Nieuwenstein

Greg Norman

Catherine J. Norris

Lauri Nummenmaa

Amy Odum

Gabriele Oettingen

Thomas M. Olino

Lindsay Oliver

Anthony D. Ong

Suzanne Oosterwijk

Leun Otten

Christopher Oveis

Nickola Overall

Romina Palermo

Anthony Papa

Jiyoung Park

Brian Parkinson

Acacia Parks

W. Gerrod Parrott

Jessica D. Payne

Mikko Peltola

Lee Pergamin-Hight

Kim Peters

Eric Peterson

Carly Peterson

K. V. Petrides

Erik Pettersson

Lauren Philbrook

Louise H. Phillips

Jared Piazza

Swann Pichon

Paul Piff

Suzanne Pineles

Richard S. Pond

Gilles Pourtois

Jay Pratt

Robert R. Provine

Samuel P. Putnam

Tom Pyszczynski

Kimberly Quinn

Eshkol Rafaeli

Anne S. Rasmussen

Kyle Ratner

Jane E. Raymond

Rebecca E. Ready

Anu Realo

Bethany C. Reeb-Sutherland

Rebecca Reed

Daniel Reisberg

Rainer Reisenzein

Michaela Riediger

Ronald E. Riggio

Maureen Ritchey

Timothy D. Ritchie

Debi Roberson

Amanda Robinson

Lucy Robinson

Rosa María Rodríguez Bailón

Christina Roecke

Neal Roese
Glenn Roisman

Ira J. Roseman

Naomi B. Rothman

Joshua Rottman

Mollie Ruben

Matthew Ruby

Melanie Rudd

Jan Rummel

James A. Russell

Magdalena Rychlowska

Andrew Ryder

Aaron Sackett

Alvaro Sanchez

Jeffrey Sanchez-Burks

Vassilis Saroglou

Wataru Sato

Blair Saunders

Krishna Savani

Susanne Scheibe

Julie A. Schermer

Antonio Schettino

Leonhard Schilbach

Brandon Schmeichel

Dominik Schoebi

Sandra Schoenfelder

Thomas W. Schubert

Marc Schulz

Sophie Scott

John G. Seamon

Paul Seli

Andrea Shafer

Stewart A. Shankman

Gary Sherman

Michelle N. Shiota

Elizabeth Shirtcliff

Carolin Showers

Patrick E. Shrout

Garriy Shteynberg

Matthias Siemer

Jen Silvers

Jeffry A. Simpson

Tamara Sims

Luke Smillie

Christine N. Smith

Gregory Smith

Jennifer Smith

Craig A. Smith

Bart Soenens

Sanjay Srivastava

Ursula M. Staudinger

Timo Stein

Marielle Stel

Danu A. Stinson

Justin L. Storbeck

Gregory P. Strauss

Margaret Stroebe

Nina Strohminger

Jenny $\mathrm{Su}$

Jordan Suchow

Amy Summerville

Gaurav Suri

Karl Szpunar 
Maya Tamir June P. Tangney

Louis Tay

Eva Telzer

Jan Theeuwes

Jason Tipples

Sascha Topolinski

Teresa Treat

Christopher Trentacosta

Ashlea Troth

Allison Troy

Joshua Tybur

Yukiko Uchida

Heather L. Urry

Andero Uusberg

Carlos Valiente
Patty Van Cappellen

Ronald van den Berg

Jan Van den Stock

Erik Van der Burg

Job van der Schalk

Jack van Honk

Henk van Steenbergen

Mascha van't Wout

Wieske Van Zoest

Marie Vandekerckhove

Eric Vanman

Carlos Velasco

Philippe Verduyn

Paul Verhaeghen

Bram Vervliet

Johan Verwoerd
Ad J. J. M. Vingerhoets

Laura Visu-Petra

Christian Wallraven

Philip C. Watkins

Ed Watkins

Christian E. Waugh

Adam Waytz

Ezra Wegbreit

Aaron C. Weidman

Mariann Weierich

Anna Weinberg

Dirk Wentura

Eric Wesselmann

Paul J. Whalen

David White

Frank H. Wilhelm

Megan L. Willis
Timothy D. Wilson

Christy Wilson-Mendenhall

Scott Wiltermuth

Piotr Winkielman

Sean Wojcik

Natalie Wyer

Dannii Yeung

Jeremy A. Yip

K. Lira Yoon

Shipstead Zachary

David H. Zald

Ren Zeelenberg

Marcel Zeelenberg

Ethan Zell

Weiwei Zhang

Michael J. Zvolensky 